\title{
Understanding Layer Promotion and Its Relationship to Melting in Krypton on Graphite Using Artificial Constraints
}

\author{
E.J. Chamberlin and M.W. Roth \\ Physics Department \\ University of Northern lowa \\ Cedar Falls, lowa 50614-0150 USA
}

Received: October 10, 2003

Accepted: January 5, 2004

\begin{abstract}
Constant particle number, density and temperature $(N, \rho, T)$ Molecular-Dynamics simulations are used to study second layer promotion and melting in a complete monolayer of krypton deposited onto a graphite substrate. In order to study the vertical behavior of the system and its relationship to melting, artificial horizontal constraints are introduced and their effects are systematically monitored. We find that horizontally confining each atom within an impenetrable cylinder increases the melting temperature $T_{m}$ and causes melting to be less dramatic. The results also suggest that there is a limiting case of there being no transition for a sufficiently small confining cylinder. Vertical excursions of the adsorbate atoms increase at the onset of melting. The system subsequently goes through a vertical transition with increasing temperature, including second layer promotion followed by extinction of the partial second layer and the presence of a sparsely populated first layer and a large population of desorbed atoms. Horizontal confinement stifles true second layer promotion, causing the atoms to spend less time in the second layer at a given temperature and resulting in a thermal blurring of the adlayer, suggesting that in-plane fluctuations are a necessary part of the layer promotion mechanism. Horizontal confinement also raises the temperature where the vertical transition occurs but does not affect its sharpness or temperature extent.
\end{abstract}

\section{INTRODUCTION}

The behavior of krypton physisorbed onto a graphite substrate has been mapped out over the last twenty years [1-16], resulting in a rich and interesting landscape of commensurate phases, sub-monolayer islands, complicated domain wall structures, solid-solid and solid-liquid phase transitions and even a re-entrant fluid. In fact, the general topic of two-dimensional (2D) and quasi-2D melting is still of scientific importance. As the accessibility to an increasing variety of nanosystems grows, adsorption of species such as krypton and other gases in confined and exotic geometries becomes realizable.

There exists a vast body of work regarding the adsorption of rare gases onto pores, which illustrates that confining geometries can strongly influence the physical behavior of an adsorbed system. As a recent example, Monte Carlo computer simulations modeling adsorption of argon (Ar) at $90 \mathrm{~K}$ in graphite pores of different shapes have been conducted [17]. There seems to be little hysteresis present in the condensation in a square-walled pore but the vertical hysteresis steps become more separated as pore width increases. The pore corners are very attractive adsorption sites and the walls are weaker; the adsorbing surface is effectively heterogeneous. The behavior of the system in the corners is very much like that of onedimensional systems.

Although there has been significant interest and effort in understanding the behavior of systems physisorbed onto spherical substrates [18-27], systems adsorbed onto Fullerenes [28-34] and even Fullerenes and their interactions [35-46], 
there has been relatively little effort placed on understanding structural and thermodynamic phase transitions of species adsorbed in confined geometries. There is a report of interesting molecular dynamics simulations of the melting transition of $r^{-12}$ repulsive particles on the surface of spheres [47]. The study focuses mainly on the order of the transition; the system is strictly $2 \mathrm{D}$ with no radial degree of freedom, and it examines various densities with 72, 122 and 272 particles. Spherical geometry requires that lattice defects be present in the solid, but the authors conclude that such a thing could work in favor of simulations on spheres and give results closer to the thermodynamic limit for much smaller systems than in planar studies because the system's relaxation is enhanced. No hysteresis loops in density through melting are observed, and the correlation length as obtained from the structure factor shows a density dependence which is concluded to be consistent with a well-known two-stage melting theory. A continuous melting transition is reported, but the system is too small to exhibit a hexatic phase. The authors believe that a hexatic phase may be seen for larger systems, and furthermore that the first-order transitions reported in planar computer simulations is completely artificial.

There are some recent and very interesting reports of computer simulations of the phases accessible to various gases placed inside [48] and adsorbed onto [49, 50] nanotube bundles. Smaller species become adsorbed within the pores and are able to exhibit order-disorder quasi-one dimensional transitions. Atoms or molecules adsorbed on the bundle exterior may, depending upon thermodynamic conditions, exist in a quasi-one dimensional "striped" phase, a quasi-two-dimensional "monolayer film" or a three-dimensional "bulk film" phase [49]. Subsequent studies of argon and krypton as well as neon, xenon and methane on nanotube bundles [50] also show that the low-pressure phase for the system is a quasi-one-dimensional phase existing within the grooves between nanotubes which can become a more complicated "three stripe" phase depending on the species followed by quasi-twodimensional and three-dimensional phases with increasing pressure.
We have investigated the behavior of argon/krypton mixtures adsorbed between two confining planes of graphite [51,52]. We found high-temperature solid phases for both argon and krypton layers partly because the adsorbate is forced into a region where the lateral corrugation from the graphite is very strong. In addition the melting temperature $T_{m}$ may be controlled quite precisely by only the slab separation and partly as a competition between vertical confinement (raising $T_{m}$ ) and the creation of in-plane room by augmentation of vertical fluctuations and ultimately coaxing of atoms away from one layer by another. As a result the melting temperature depends strongly on the plane spacing in a certain range. In addition, due to the different properties of argon and krypton, we observe almost pure component extraction for certain temperatures and slab spacings. In addition, argon monolayers are forced into commensurate and rotated phases, the former of which could be accessible over a wide temperature range.

Clearly, confinement of adsorbed species can result in system-specific, surprising and potentially useful results. The effects of many types of confinement on the behavior of adsorbed systems have been studied, and the purpose of this work is to extend the current body of knowledge by examining krypton monolayers that are deposited onto a graphite substrate where each atom is confined horizontally by a hard cylindrical wall. Although the type of confinement chosen in this work does not have a direct physical analogue, it does exhibit the symmetry, scale and long-range limit that real confinement (such as an array of nanotubes) would impose, and is therefore physically meaningful. The point of the study at hand is not to model a new system but to examine the effect of selective constraints on the model of a real system, and to show what can be gained by such an effort. The study at hand is motivated by a desire to better understand the behavior of out-of-plane (vertical) atomic fluctuations with differing temperature as well as their role in phase transitions. In addition we hope to better understand any anisotropic character of the systems behavior, where its vertical character is markedly different from its in-plane character. The type of confinement used here provides 
considerable insight into the relationship of in-plane and out-of-plane dynamics for physisorbed systems, and into the mechanism of layer promotion.

\section{COMPUTATIONAL APPROACH}

The method utilized in this study is a constant particle number, planar density and temperature $(\mathrm{N}, \rho, \mathrm{T})$ Molecular-Dynamics (MD) method with free boundary conditions in the vertical direction and periodic boundary conditions in the $(x, y)$ plane. The computational cell is constructed such that the adlayer is complete ( $\rho=1$ in units of 0.0636 atoms per square Angstrom) for $\mathrm{N}=400$. The atoms are initially placed at registered $\sqrt{3} X \sqrt{3} R 30^{\circ}$ positions in the $(\mathrm{x}, \mathrm{y})$ plane, each one inside its individual confining cylinder of radius $\mathrm{R}$ centered at each registry position. Furthermore, each atom is placed close to its vertical equilibrium height. A standard Verlet algorithm is used to integrate the equations of motion and the temperature is held constant by velocity rescaling. Typical runs are equilibrated for $10^{4}$ time steps and averages taken over $6 \times 10^{4}-10^{5}$ steps using an interval $\Delta t=4$ femtoseconds (fs).

A central component of the experiment is the horizontal confinement of the krypton through krypton-cylinder interactions. The type of confinement which is applicable to all of the results presented here is "hard confinement". Here, cylinders of radius $\mathrm{R}$ are placed around every atom and are centered around the atoms' initial registry points. Simulations are conducted with $R=1,1.25,1.5,1.75$ and $2 \AA$, as well as for $R=\infty$ (the unconfined case). $R>2 \AA$ is not utilized because in such a case there would be overlap which is inconsistent even within the context of the simulation; the hard walls would be perfectly permeable for some atoms and perfectly reflecting for others. As the simulation proceeds, an atom's velocity is reflected when its center of mass hits the cylindrical wall, and it is re-positioned if it is slightly outside the cylinder.

There are two other interactions which are important in the simulations: krypton-krypton and krypton-substrate. We employ a three-dimensional orthogonal Cartesian co-ordinate system, with the $x$ axis in the $\Gamma-K$ direction of the graphite lattice and the z-axis (the vertical coordinate) perpendicular to the graphite basal plane. The krypton-krypton potential is of the Aziz form,

$$
\begin{aligned}
& V_{i j}^{*}\left(x_{i j}\right)=B\left(x_{i j}\right)^{\gamma}- \\
& F\left(x_{i j}\right)\left[C_{6}\left(x_{i j}\right)^{-6}+C_{8}\left(x_{i j}\right)^{-8}+C_{10}\left(x_{i j}\right)^{-10}\right]
\end{aligned}
$$

and details are given elsewhere [53]. In equation (1) $x_{i j}=r_{i j} / r_{m}$ where $r_{i j}$ denotes the center-of-mass distances between Krypton atoms (i) and (j)), $V_{\mathrm{ij}}\left(\mathrm{x}_{\mathrm{ij}}\right)=\varepsilon_{\mathrm{Kr}} \mathrm{V}^{*}{ }_{\mathrm{ij}}\left(\mathrm{x}_{\mathrm{ij}}\right)$ and the function $F\left(x_{i j}\right)$ is given by

$$
F\left(x_{i j}\right)=\left\{\begin{array}{c}
e^{-\left[\left(\frac{D}{x_{i j}}\right)\right]^{2}}, \quad x_{i j}<D . \\
1, \quad x_{i j} \geq D
\end{array}\right.
$$

Equation 2 describes screening of the dispersion term of the potential. Table 1 contains the potential parameters $\left(\varepsilon_{\mathrm{Kr}}, r_{\mathrm{m}}, \mathrm{B}\right.$, $\left.\alpha, \mathrm{\gamma}, \mathrm{C}_{6}, \mathrm{C}_{8}, \mathrm{C}_{10}, \mathrm{D}\right)$ for Krypton.

The Krypton-Graphite interactions were calculated using Steele's Fourier expansion:

$$
U_{i}=E_{0 i}\left(z_{i}\right)+\sum_{n=1}^{\infty} E_{n i}\left(z_{i}\right) f_{n i}\left(x_{i}, y_{i}\right),
$$

with

$$
E_{o i}\left(z_{i}\right)=\frac{2 \pi q \varepsilon \sigma^{6}}{a_{s}}\left(\frac{2 \sigma^{6}}{45 d\left(z_{i}+0.72 d\right)^{9}}+\frac{2 \sigma^{6}}{5 z_{i}^{10}}-\frac{1}{z_{i}^{4}}-\frac{2 z_{i}^{2}+7 z_{i} d+7 d^{2}}{6 d\left(z_{i}+d\right)^{5}}\right)
$$




$$
E_{n i}\left(z_{i}\right)=\frac{2 \pi \varepsilon \sigma^{6}}{a_{s}}\left\{\left(\frac{\sigma^{6}}{30}\right)\left(\frac{g_{n}}{2 z_{i}}\right)^{5} K_{5}\left(g_{n} z_{i}\right)-2\left(\frac{g_{n}}{2 z_{i}}\right)^{2} K_{2}\left(g_{n} z_{i}\right)\right\}
$$

The series in equation (3) utilizing the terms shown in equations (4) and (5) converges rapidly so only the $n=1$ term containing

$$
f_{1}\left(x_{i}, y_{i}\right)=\left.f_{n}\left(x_{i}, y_{i}\right)\right|_{n=1}=-2\left\{\cos \frac{2 \pi}{a}\left(x+\left(\frac{y}{\sqrt{3}}\right)\right)+\cos \frac{2 \pi}{a}\left(x-\left(\frac{y}{\sqrt{3}}\right)\right)+\cos \frac{4 \pi}{a}\left(\frac{y}{\sqrt{3}}\right)\right\} \text {. }
$$

is retained. All the important potential parameters for krypton-graphite interactions are identified in Table 1.

\begin{tabular}{|c|c|}
\hline Parameter & Value \\
\hline$\varepsilon_{\mathrm{Kr}}$ & $199.9 \mathrm{~K}$ \\
\hline $\mathrm{r}_{\mathrm{m}}$ & $4.012 \AA$ \\
\hline $\mathrm{B}$ & $1.215312 \times 10^{7}$ \\
\hline$\alpha$ & 16.496763 \\
\hline$\gamma$ & 2.40 \\
\hline $\mathrm{C}_{6}$ & 1.1561739 \\
\hline $\mathrm{C}_{8}$ & 0.5414923 \\
\hline $\mathrm{C}_{10}$ & 0.2839735 \\
\hline $\mathrm{D}$ & 1.28 \\
\hline$\varepsilon$ & $64.83 \mathrm{~K}$ \\
\hline$\sigma$ & $3.22 \AA$ \\
\hline $\mathrm{d}$ & $3.37 \AA$ \\
\hline $\mathrm{a}_{\mathrm{s}}$ & $5.24 \AA^{2}$ \\
\hline $\mathrm{q}$ & 2 \\
\hline
\end{tabular}

Table 1. Kr-Kr and $\mathrm{Kr}$-graphite potential parameters from reference [53].

\section{RESULTS AND DISCUSSION}

\section{a. In-Plane Behavior (Melting)}

As temperature increases in the solid phase, the first transition exhibited by the system is melting; several structural and energetic quantities are useful in delineating such a transition.
The order parameter OP1 is defined

as

$$
O P 1=\frac{1}{6 N} \sum_{i=1}^{N}\left\langle\sum_{s=1}^{6} e^{i \vec{g}_{s} \cdot \bullet_{i}}\right\rangle
$$

where the $\vec{g}_{s}$ are the six reciprocal lattice vectors for the graphite substrate and the brackets denote a thermal average. OP1 is equal to unity for a zero-temperature commensurate lattice, and vanishes, for example, in the cases of ideal infinite incommensurate lattices or for systems that uniformly sample the $(x, y)$ plane due to thermal fluctuations. Although OP1 gives information about the atomic positional order with respect to the substrate, it is possible to have a low value for OP1 but have considerable in-plane collective motion of the adsorbate. The order parameter OP2, defined as

$$
O P 2=\frac{1}{3 N(N-1)} \sum_{i=1}^{N} \sum_{j>1}^{N}\left\langle\sum_{s=1}^{6} e^{1 \vec{g}_{s} \cdot \vec{r}_{i j}}\right\rangle
$$

helps to further characterize melting, as it gives information about the over-layer itself. In equation (8), $\vec{r}_{i j}$ is the displacement vector from atom (i) to atom (j), so the krypton lattice vectors are being compared to those for a commensurate $\sqrt{3} X \sqrt{3} R 30^{\circ}$ lattice. So for commensurate systems such as $\mathrm{Kr}$ on graphite, the order parameters OP1 and OP2 defined in equations (7) and (8) are excellent indicators of translational order and how that order changes through melting. The parameters OP1 and OP2 are shown as functions of temperature for various confining radii in Figure 1.

In addition the thermal average $\left\langle E_{1} f_{1}\right\rangle$ of the Steele corrugation term in the krypton-graphite potential (referred to as 
$\left.\left\langle E_{1}\right\rangle\right)$ is a reliable indicator of registry as well as melting. In a zero-temperature commensurate lattice, $\left\langle E_{1}\right\rangle$ is equal to about $40 \mathrm{~K}$ but its value decreases with increasing thermal fluctuations, approaching zero for uniform or random sampling of the $(x, y)$ plane. Calculated values of $\left\langle\mathrm{E}_{1}\right\rangle$ are shown as functions of temperature for various confining radii in Figure 2 . It is apparent that confinement causes the melting temperature to increase and the transition itself to become less dramatic. It is interesting to note that for smaller values of $R$ the value of the order parameters and $\left\langle E_{1}\right\rangle$ do not go to zero at high temperatures, indicating the presence of a registered fluid. In conjunction with the behavior of the melting temperature $T_{m}$ with decreasing $R$ shown in Table 2, it seems that the melting transition approaches some maximum value where the solid and fluid lose their distinction.

In most melting studies, OP1 and OP2 yield identical results, which is to say that translational order with respect to the substrate is lost along with the translational order of the lattice itself. It is noteworthy that, under horizontal confinement, the values of OP2 for $R=1.75 \AA$ and $2 \AA$ are systematically higher than zero while the corresponding values of OP1 vanish within the calculated uncertainty. Such results are suggestive that there is some degree of cooperative krypton-krypton motion present, due to the horizontal confinement.
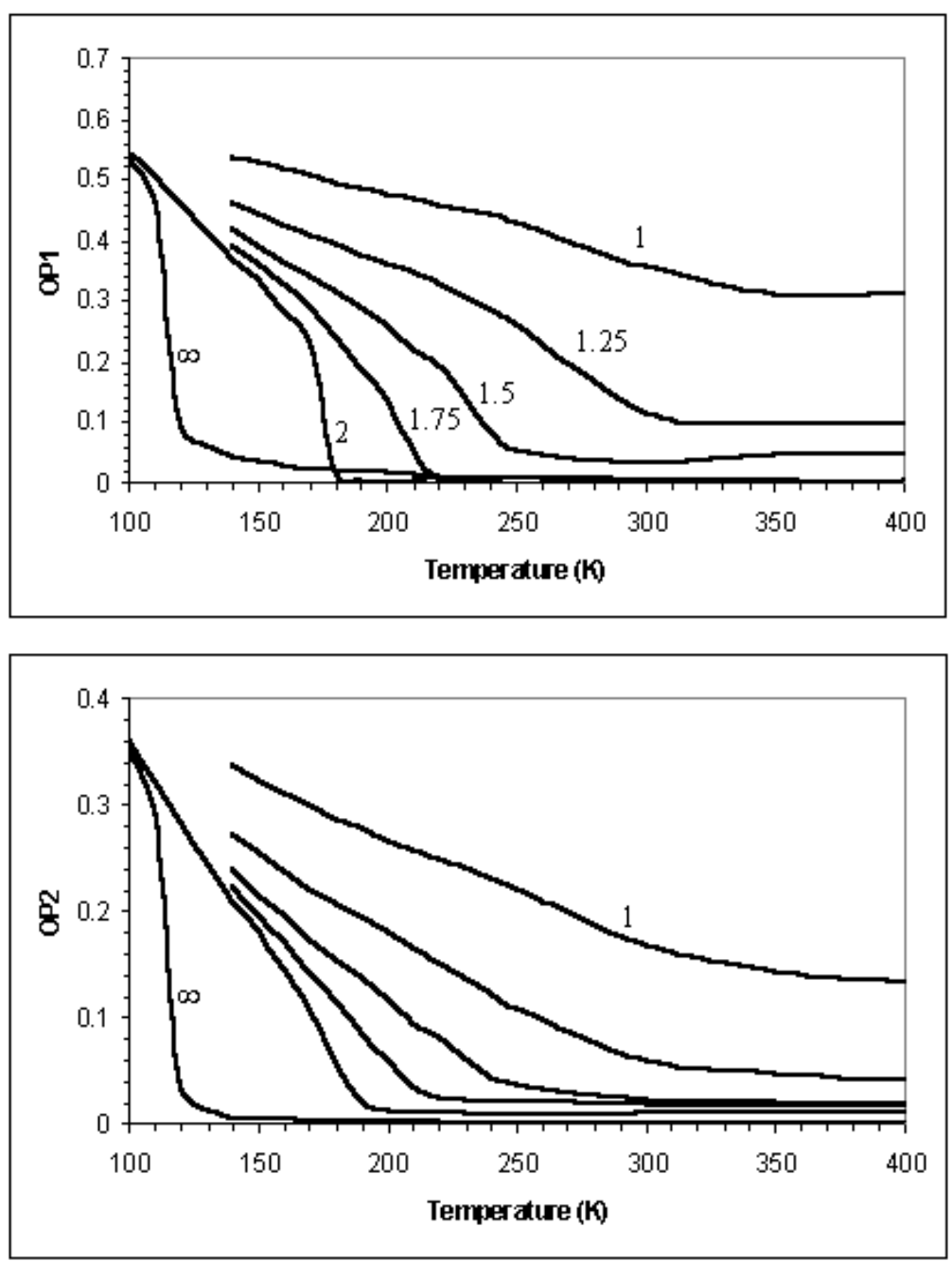

Figure 1. Order parameters OP1 (top) and OP2 (bottom) as functions of temperature, for various confining radii $\mathrm{R}$, as indicated on the plots. Only the limiting confining radii are indicated on the lower plot. 


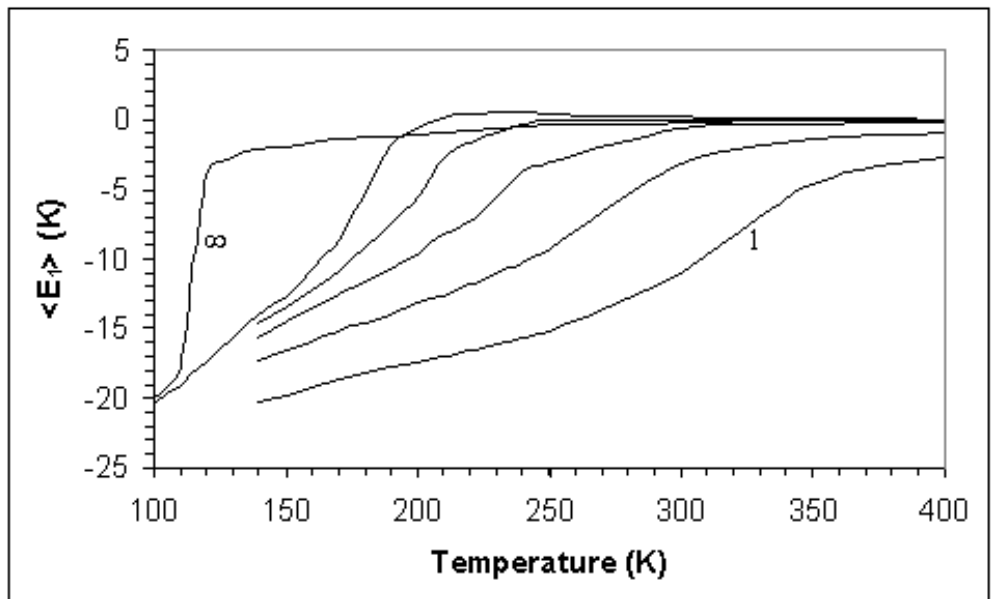

Figure 2. Thermal average $\left\langle\mathrm{E}_{0}\right\rangle$ of the lateral corrugation in the adsorbate-substrate Steele interaction as functions of temperature for various confining radii $R$ as indicated on the graph. Format is the same as for the bottom plot in Figure 1.

\section{b. Out-Of-Plane (Vertical) Behavior}

Some thermodynamic parameters are helpful in delineating the vertical dynamics of the system. The thermal average of the krypton-krypton interaction per atom $\left\langle\mathrm{U}_{\mathrm{Kr}-\mathrm{Kr}}\right\rangle$ is given by

$$
\left\langle U_{K r-K r}\right\rangle=\frac{1}{N}\left\langle\sum_{i=1}^{N} \sum_{j=i+1}^{N} V_{i j}\right\rangle
$$

$\left\langle\mathrm{U}_{\mathrm{Kr}-\mathrm{Kr}}\right\rangle$ is useful in determining the nature and location of a phase transition involving changes in the translational order of the monolayer. Usually, rapid changes in krypton-krypton energy comes from either enhanced thermal fluctuations and/or changes in the adlayer topology. The thermal average of the laterally averaged Steele energy $\left\langle E_{0}\right\rangle$ per atom is given by

$$
\left\langle E_{0}\right\rangle=\frac{1}{N}\left\langle\sum_{i=1}^{N} E_{0 i}\right\rangle
$$

Since the laterally averaged energy $E_{0 \mathrm{i}}$ is dependent only on the height of atom (i) above the graphite basal plane, $\left\langle\mathrm{E}_{0}\right\rangle$ is an excellent indicator of the vertical dynamics of the adlayer. In conjunction with other parameters, $\left\langle E_{0}\right\rangle$ can signal the rapid increase of individual atomic vertical fluctuations (including the limiting case of desorption) or it could also signal collective

\begin{tabular}{|c|c|c|c|}
\hline $\mathbf{R}(\mathbf{A})$ & $\mathbf{T}_{\mathbf{m}} \mathbf{( K )}$ & $\Delta \mathbf{T}_{\mathbf{z}}(\mathbf{K})$ & $\mathbf{T}_{\text {shoulder }}(\mathbf{K})$ \\
\hline $\mathbf{1}$ & 271 & $250-350$ & 300 \\
\hline $\mathbf{1 . 2 5}$ & 266 & $250-300$ & $*$ \\
\hline $\mathbf{1 . 5}$ & 231 & $220-300$ & 250 \\
\hline $\mathbf{1 . 7 5}$ & 205 & $200-300$ & $*$ \\
\hline $\mathbf{2}$ & 174.5 & $170-250$ & 190 \\
\hline $\boldsymbol{\infty}$ & 115 & $110-250$ & 150 \\
\hline
\end{tabular}

Table 2. Melting temperature $T_{m}$, range in temperature $\Delta T_{z}$ over which the vertical character of the systems most rapidly change and the temperature $\mathrm{T}_{\text {shoulder }}$ at which the atomic height distributions in Figures 3-6 show a maximum shoulder near where a second layer would appear. The unconfined system actually does support a partial second layer. Uncertainties in $T_{m}$ range from about $\pm 10 \mathrm{~K}$ at $\mathrm{R}=1 \AA$ to $\pm 3 \mathrm{~K}$ at $R=\infty$. Entries with asterisks $\left(^{*}\right)$ are for values of $R$ not shown in Figures 3-6.

vertical motion of the system. Lastly, the thermal average of the total internal energy $U$ per atom is given by

$$
\langle U\rangle=\frac{1}{N}\left\langle\sum_{i=1}^{N} U_{i}+\sum_{i=1}^{N} \sum_{j=i+1}^{N} V_{i j}\right\rangle+1.5 k_{B} T
$$


and is a standard and useful parameter in monitoring phase transitions because it depends on the system's' total configurational energy as well as its temperature. Because we utilize MD, which is force-driven, we do not include continuum corrections in our calculations.
$\left\langle\mathrm{U}_{\mathrm{Kr}-\mathrm{Kr}}\right\rangle,\left\langle\mathrm{E}_{0}\right\rangle$ and $\langle\mathrm{U}\rangle$ are shown as functions of temperature for various confining radii in figure 3 , and it is apparent that there is a temperature region where they change most rapidly, suggesting a phase transition. Since the krypton-krypton energy per particle exhibits the same
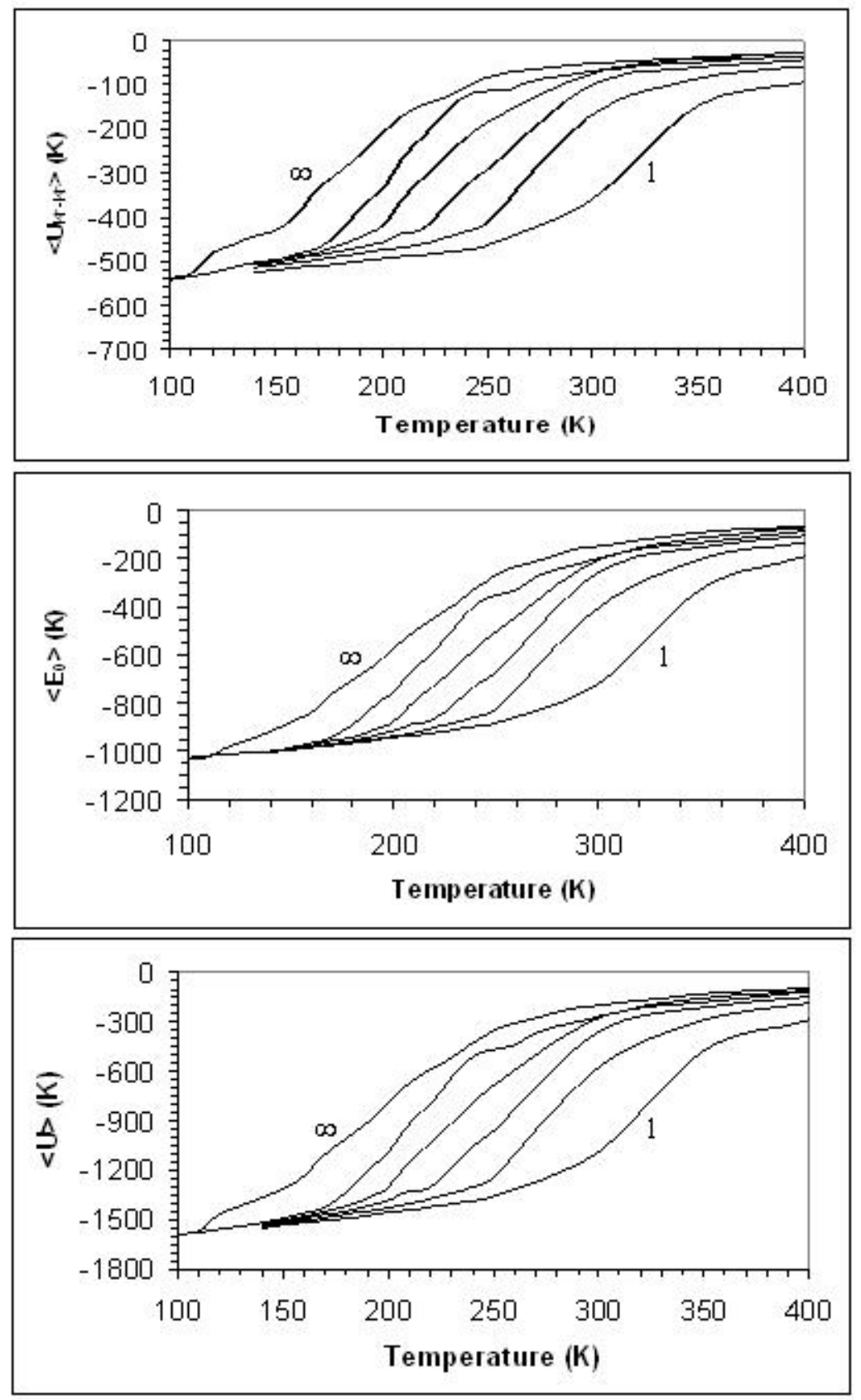

Figure 3. Thermal averages of the krypton-krypton interaction, laterally averaged component of the Steele energy and total internal energy as functions of temperature for various confining radii R. Format is the same as for the bottom plot in Figure 1. 

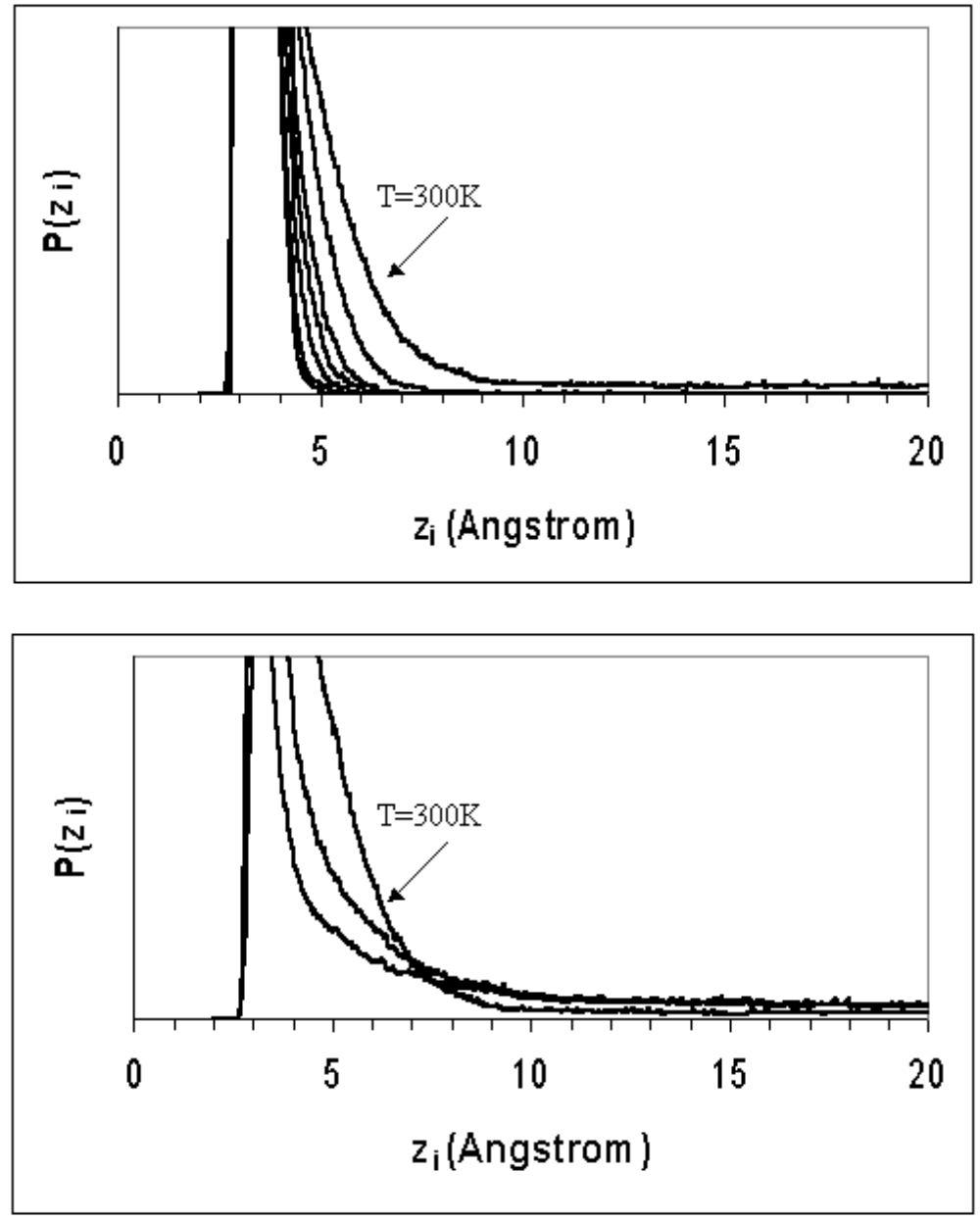

Figure 4. Atomic height distributions $P\left(z_{i}\right)$ at various temperatures ranging from $T=140 \mathrm{~K}$ to 300 $\mathrm{K}$ (top) and $\mathrm{T}=300 \mathrm{~K}$ to $400 \mathrm{~K}$ (bottom) for a confining radius $\mathrm{R}=1 \AA$. The first-layer upper peaks broaden with increasing temperature up until $\mathrm{T}_{\text {shoulder }}=300 \mathrm{~K}$ is reached in the upper plot but behave oppositely in the lower plot. The vertical scale is arbitrary but identical to those in Figures 5-7 and temperature ranges are chosen so as to provide a reasonable visual perspective.

signature as the other two quantities, collective motion involving migration of the monolayer is ruled out. The approximate temperature ranges $\Delta T_{z}$ of the most rapid changes of $\left\langle\mathrm{U}_{\mathrm{Kr}-\mathrm{Kr}}\right\rangle,\left\langle\mathrm{E}_{0}\right\rangle$ and $\langle\mathrm{U}\rangle$ are shown in Table 2, and inspection of $T_{m}$ and $\Delta T_{z}$ reveals that melting and the onset of increasing vertical fluctuations happen at the same temperatures. It is interesting to note how horizontal confinement affects the vertical behavior of the system. The sharpness and temperature duration of the transition are nearly unaffected, but the temperature location increases with decreasing confining cylinder radius $R$.
Such a result suggests that the coupling of vertical and horizontal fluctuations plays a significant role in the dynamics of adsorbed systems. The atomic height distributions $\mathrm{P}\left(\mathrm{z}_{\mathrm{i}}\right)$ provide structural information about the vertical behavior of the system and place the thermodynamic results in broader context. The height distribution is constructed by calculating the frequency of occurrence of the height of each atom (i). Height distributions at various temperatures for $\mathrm{R}=$ $1 \AA, 1.5 \AA, 2 \AA$ and $\infty$ are shown in Figures 4-7, respectively. An interesting feature common to systems for all confining radii is that, starting in the solid phase, the 

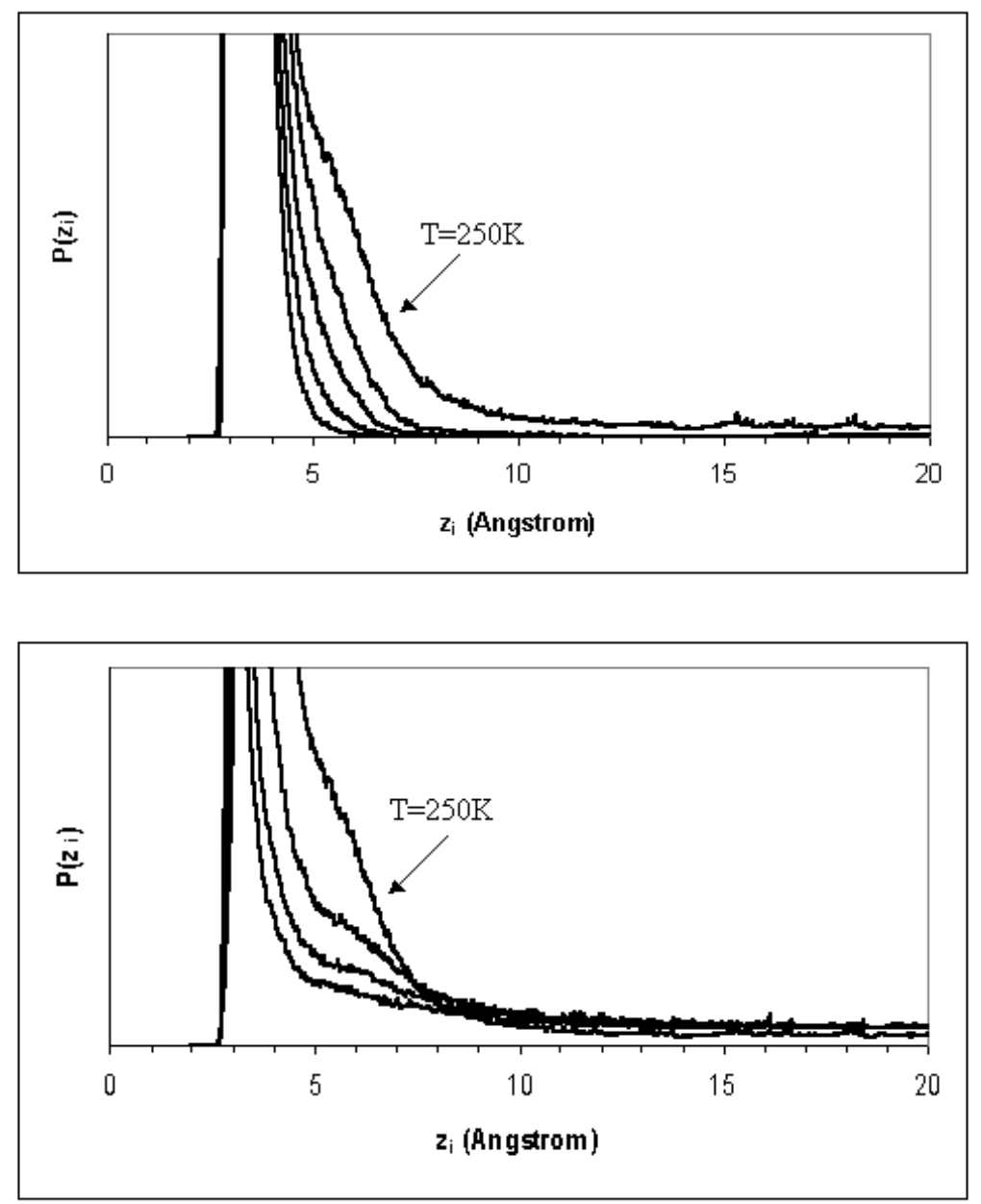

Figure 5. Atomic height distributions $P\left(z_{i}\right)$ at various temperatures ranging from $T=150 \mathrm{~K}$ to 250 $\mathrm{K}$ (top) and $\mathrm{T}=250 \mathrm{~K}$ to $400 \mathrm{~K}$ (bottom) for a confining radius $\mathrm{R}=1.5 \AA$. The format is the same as in Figure 4 except that $\mathrm{T}_{\text {shoulder }}=250 \mathrm{~K}$.

distribution begins to lift where a second layer's height would be, indicating a larger population of atoms at that height. The degree of lifting increases up to a certain temperature designated as $\mathrm{T}_{\text {shoulder }}$ where there is maximum lifting and a "shoulder" appears on the distributions. For strongly confined monolayers there is almost no true shoulder and for unconfined systems the "shoulder" is actually a second peak, suggesting that unconfined systems support second layer promotion but confining the system stifles the separation of the second layer from the first because the adsorbate spends less time in promotion. Hence, increased confinement causes a temperature-induced blurring of the adlayer as opposed to true second layering. Subsequently, as temperature increases the shoulder (as well as the prominent monolayer peak) drops and the tail of the distribution (the distribution for heights greater than where the second layer is) lifts from zero, suggesting that the second layer is extinguished in favor of a low density first layer and a considerable population of desorbed atoms. Inspection of the values of $\mathrm{T}_{\text {shoulder }}$ shown in Table 2 shows that maximum second layer promotion occurs after melting and well within the range of the vertical transition. In fact, inspection of figure 3 confirms that $T_{\text {shoulder }}$ coincides with the most rapid change in thermodynamic parameters sensitive to vertical fluctuation. 

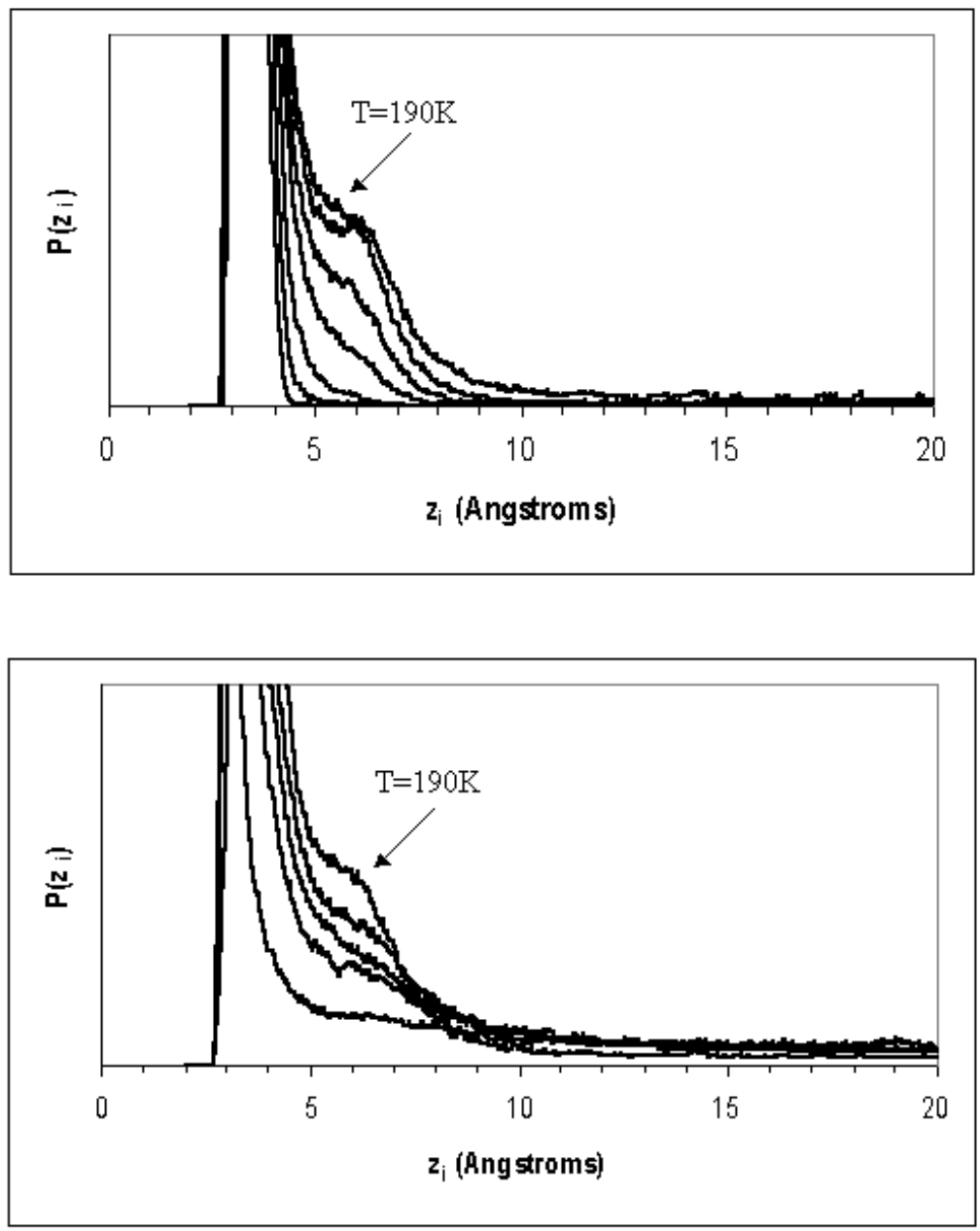

Figure 6. Atomic height distributions $\mathrm{P}\left(\mathrm{z}_{\mathrm{i}}\right)$ at various temperatures ranging from $\mathrm{T}=110 \mathrm{~K}$ to 190 $\mathrm{K}$ (top) and $\mathrm{T}=190 \mathrm{~K}$ to $370 \mathrm{~K}$ (bottom) for a confining radius $\mathrm{R}=2 \AA$. The format is the same as in Figure 4 except that $\mathrm{T}_{\text {shoulder }}=190 \mathrm{~K}$.

Hence a picture emerges where the monolayer melts, then undergoes a transition in vertical character which involves second layer promotion, followed by extinction of the second layer ultimately resulting in a sparsely populated first layer and frequent desorption.

As a final remark, it should be remembered that our model is a classical one and as a result any quantum effects from confinement are not included. Although the results presented here for the smallest values of $R$ help establish trends and aid in the understanding of the system dynamics, any realistic model of the system in such a strongly confined regime should incorporate quantum effects which may be non- negligible. In addition, experimental work related to our calculations would be highly beneficial and desirable. It may be possible to approximate our hard cylinder confinement with a pore system capped by a graphite substrate or perhaps with some carefully designed nanostructure

\section{CONCLUSIONS}

The major conclusions of this work are as follows:

i. Pronounced horizontal confinement of krypton within individual cylinders results in increased melting temperatures $T_{m}$ and dulling of the melting transition. 

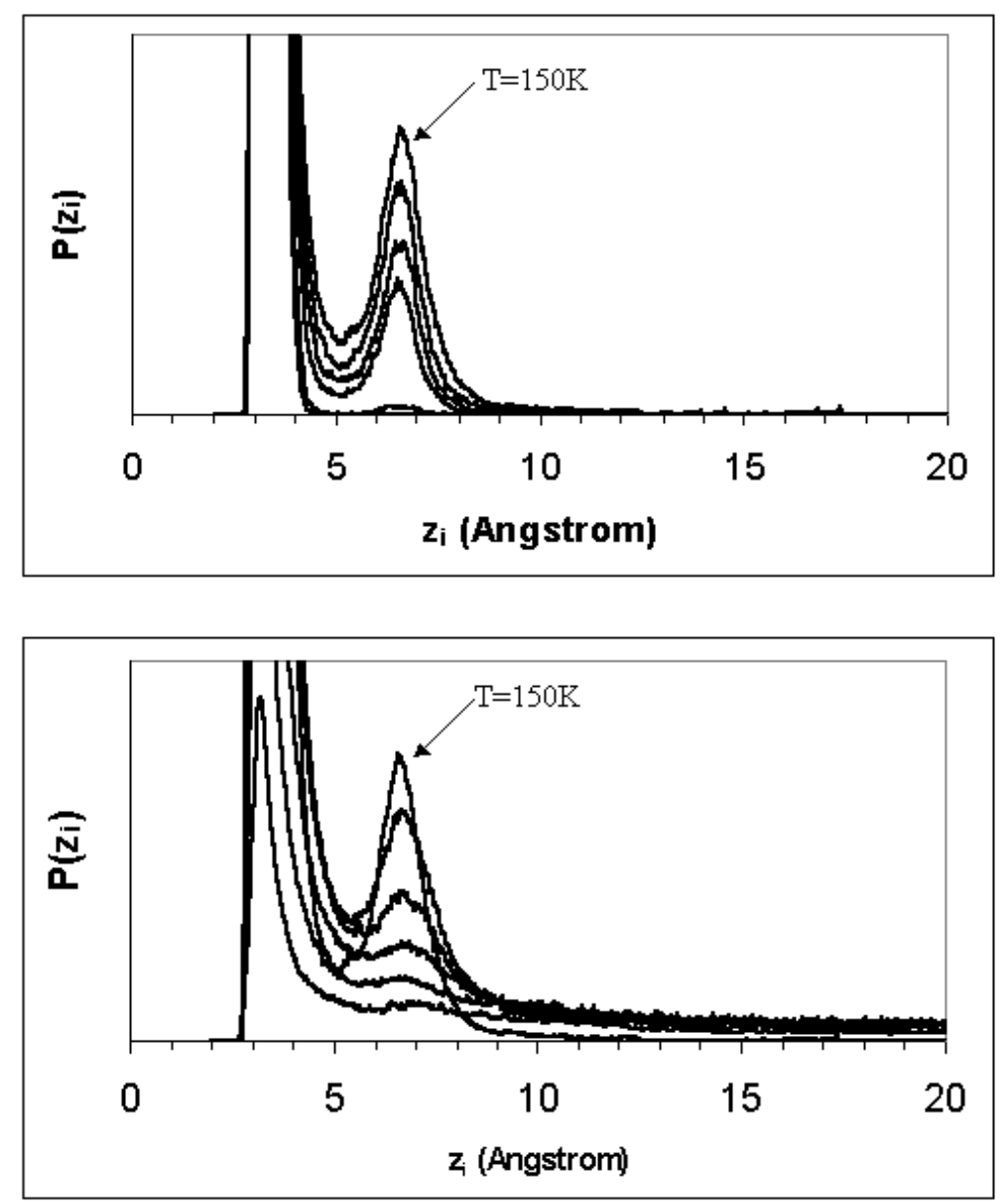

Figure 7. Atomic height distributions $P\left(z_{i}\right)$ at various temperatures ranging from $T=140 \mathrm{~K}$ to 150 $\mathrm{K}$ (top) and $\mathrm{T}=150 \mathrm{~K}$ to $400 \mathrm{~K}$ (bottom) for a confining radius $\mathrm{R}=\infty$. The format is the same as in Figure 4 except that $\mathrm{T}_{\text {shoulder }}=150 \mathrm{~K}$.

ii. The melting temperature $T_{m}$ reaches a constant value with pronounced confinement and behavior of the order parameters suggests that the solid and fluid would lose their distinction as $R$ decreases past a certain value.

iii. There appears to be vertical transition exhibited by the monolayers which begins with melting and lasts for about 100K. It involves second layer promotion followed by extinction of the second layer and subsequently a sparsely populated first layer and many desorbed atoms.

iv. Pronounced horizontal confinement raises the temperature at which the vertical transitions take place but does not affect either its temperature extent or its sharpness.

v. The nature of second layer promotion is affected by horizontal confinement. Pronounced confinement causes the adsorbate atoms to spend less time in true second layer promotion in favor of briefer excursions resulting in thermal blurring of the adlayer.

vi. Using constraints which have no exact physical analogue can lend considerable insight into understanding the dynamics and phase transitions of real systems. 


\section{ACKNOWLEDGEMENTS}

The authors are grateful to the University of Northern lowa for its support with a SOAR grant.

\section{REFERENCES}

1. N.D. Shrimpton, M.W. Cole, and W.A. Steele, in Phase Transitions in Surface Films, Ed. H. Taub, G. Torzo, H. Lauter, and S.C. Fain, (Plenum, New York, 1991)

2. E. D. Specht, A. Mak, C. Peters, M. Sutton, and R. J. Birgenau, K. L. D'Amico and D.E. Moncton, S. E. Nagler and P. M. Horn, Zeit. Phys. B, $\underline{69}, 347$ (1987).

3. D. M. Butler, J. A. Litzinger and G. A. Stewart, Phys. Rev. Lett. 44, 466 (1980).

4. R. J. Birgenau, E. M. Hammons, P. Heiney and P. W. Stephens, Ordering in Two Dimensions, Elsevier North Holland Publishing, 29 (1980).

5. S. Chung, A. Kara, J. Z. Larese, W. Y. Leung and D. R. Frankl, Phys. Rev. B, 35, 4870 (1987).

6. M.D. Chinn and S. C. Fain, Jr., Phys. Rev. Lett. 39, 146 (1977).

7. C. A. Guryan, K. B. Lee and P. W. Stephens, A. I. Goldman and J. Z. Larese, P. A. Heiney and E. Fontes, Phys. Rev. B, 37, 3461 (1988).

8. R. Hoja and R. Marx, Phys. Rev. B 34, 7823 (1986).

9. A. Inaba, J. A. Morrison and J. M. Telfer, Mol. Phys. 162, 961 (1987).

10. N. Dupont-Pavlovsky, C. Bockel and A. Thomy, Surf. Sci. 160, 12 (1985).

11. Y. Larher and A. Terlain, J. Chem. Phys. 72, 1052 (1980)

12. T.P. Vo and T. Fort, Jr., J. Phys. Chem. 91, 6638 (1987).

13. J. A. Venables, J. L. Seguin, J. Suzanne and M. Bienfait, Surf. Sci., 145, 345 (1984).

14. T. H. Ellis, G. Scoles and U. Valbusa and $\mathrm{H}$. Jónsson and J. H. Weare, Surf. Sci., 155, 499 (1985).

15. R. M. Suter and N. J. Colella, R. Gangwar, Phys. Rev. B 31, 627 (1985).

16. K.J. Niskanen and R. B. Griffiths, Phys. Rev. B 32, 5858 (1985).

17. Mary J. Bojan and William A. Steele, Carbon 361417 (1998).
18. Jouko Kankare and Igor A. Vinokurov, Langmuir 155591 (1999).

19. P.J. Upton, J.O. Indekeu, and J.M. Yeomans, Phys Rev B 40666 (1989).

20. I.A. Hadjiagapiou, J. Phys. Chem. B. 10189 (1997).

21. Ioannis Hadjiagapiou, J. Chem. Phys. 1052927 (1996).

22. A. Hanke and S. Dietrich, Phys. Rev. E 595081 (1999).

23. Z. Adamczyk and P. Belouschek, Journal of Colloid and Interface Science, 146123 (1991).

24. Y.L. Lee, W.S. Chou and L.H. Chen, Surf. Sci, 414363 (1998).

25. O.N. Stavinskaya, N.I. Shklovskaya and V.K. Imshennik, Russ. J. App. Chem. 68 (10 p 1), 1416 (1991).

26. M. Wada, S. Uda, and M. Kato, Philos. Mag. A 5931 (1989).

27. M. Wada, A. Fujii, and T. Yoshida, Philos. Mag B 64485 (1991).

28. H.W. Kroto, J.R. Heath, S.C. O'Brien, R.F. Curl and R.E. Smalley, Nature 318 162 (1985).

29. W. Kratschmer, J. Rathousky and A. Zukal, Carbon 37301 (1999).

30. A. Martinez-Alonso, J.M.D. Tascon and E.J. Bottani, J Phys. Chem .B 105135 (2001).

31. Vladimir Yu Gusev, Sigrid Ruetsch and llya E. Popeko, J Phys. Chem. B 103 6498 (1999).

32. K. Kaneko, C. Ishii, T. Arai and $\mathrm{H}$. Suematsu, J. Phys. Chem. 976764 (1993).

33. A. Martínez-Alonso, J.M.D. Tascón and E.J. Bottani, Langmuir 161343 (2000).

34. M. Fastow, Y. Kozirowski and $M$. Folman, Surf. Sci. 331121 (1995).

35. M. Folman, M. Fastow and $Y$. Kozirowski, Langmuir 131118 (1997).

36. C.R. Reid, I.P. O'koye and K.M. Thomas, Langmuir 142415 (1998).

37. C. Girard, P. Lambin, A. Dereux, and A. A. Lucas, Phys. Rev. B 4911425 (1994).

38. W. Krätschmer, K. Fostiropoulos and D.R. Huffman, Chem. Phys. Lett. 170 167 (1990).

39. C. Rey, L.J. Gallego, and J.A. Alonso, Phys. Rev. B 498491 (1994).

40. Seong Gon Kim and David Tomanek, Phys. Rev. Lett. 722418 (1994).

41. I.M.K. Ismail and S.L. Rodgers, Carbon, 30229 (1992). 
42. Jerry Cioslowski and Eugene D. Fleischmann, J. Chem. Phys. 943730 (1991).

43. D.S. Bethune, R.D. Johnson and J.R. Salem, Nature 366123 (1993).

44. Martin Saunders, Hugo A. JimenezVazquez, and R. James Cross, J. Am. Chem. Soc. 116, 2193 (1994).

45. D.E. Giblin, M.L. Gross, and R.J. Cross, J. Am. Chem. Soc. 119, 9883 (1997).

46. T. Braun, H. Rausch, Koblar Jackson, Efthimios Kaxiras and Mark R. Pederson, Chem. Phys. Lett. 237, 443 (1995).

47. A. Perez-Garrido and M.A. Moore, Phys. Rev. B 589677 (1998).
48. M. Mercedes Calbi, Silvina M. Gatica, Mary J. Bojan, George Stan and Milton W. Cole, Rev. Mod. Phys. 73857 (2001).

49. S.M. Gatica, M.J. Bojan. G. Stan and M.W. Cole, J. Chem. Phys, 1143765 (2001).

50. M. Mercedes Calbi, Silvina M. Gatica, Mary J. Bojan and Milton W, Cole, J. Chem. Phys. 1159975 (2001).

51. K. Bader and M.W. Roth, Am. J. Undergrad. Res. 1(1), 9 (2002).

52. K. Bader and M.W. Roth, Surf. Sci., 538 (1-2) 30 (2003).

53. R. A. Aziz, Mol. Phys. 38, 177 (1979)
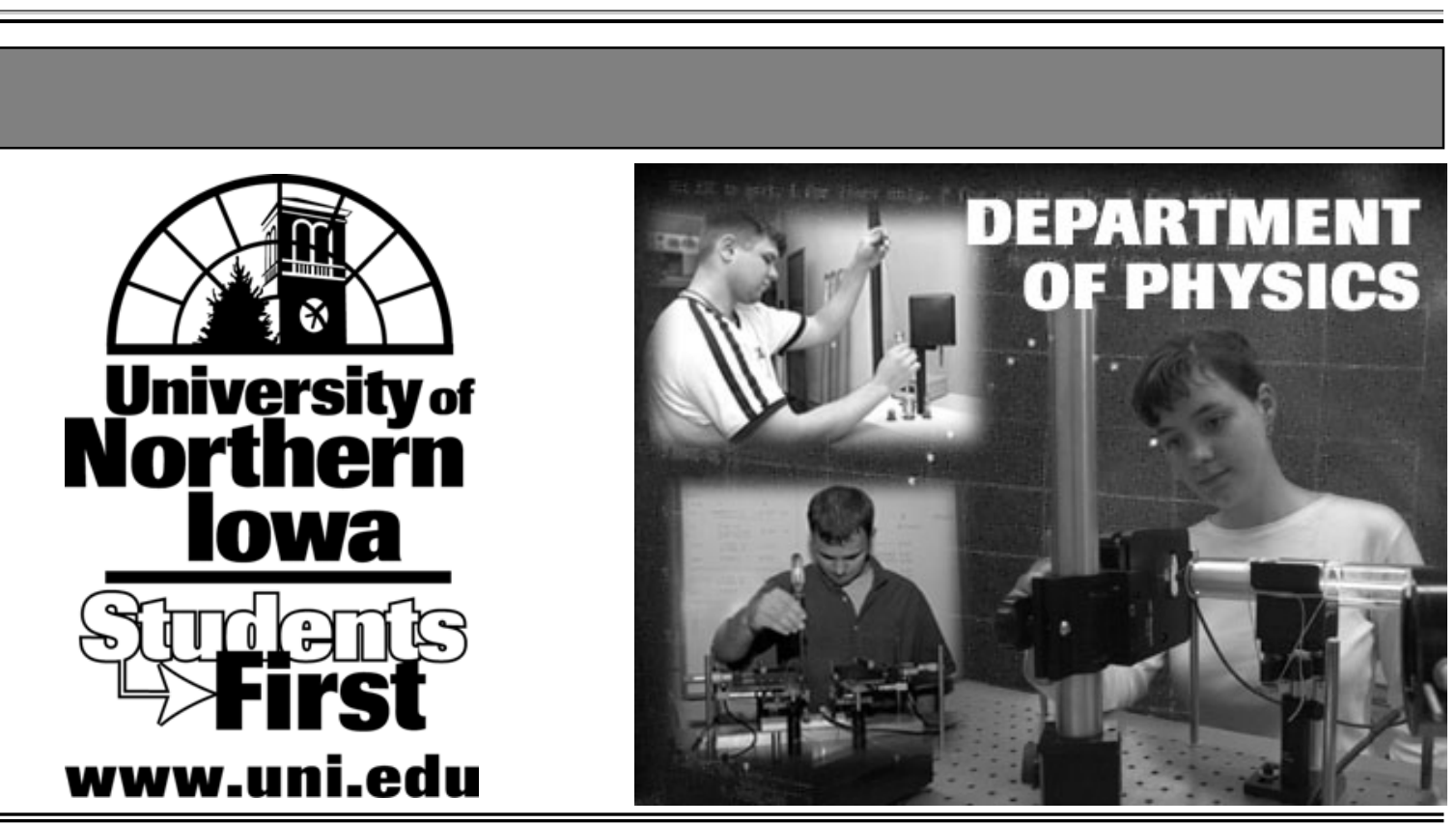

\section{Subscribe to the American Journal of Undergraduate Research}

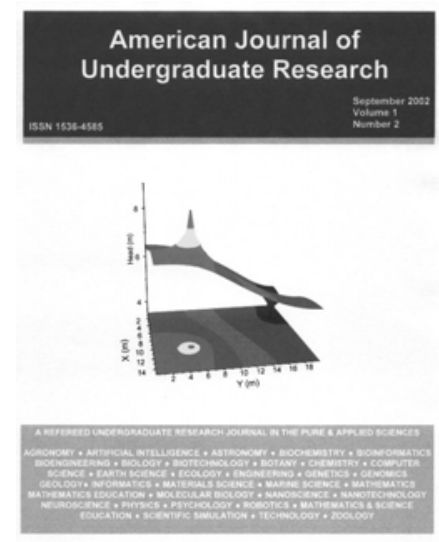

Individual Rate:

\$20/year

Institutional Rate: \$40/year

Back Issues available at $\$ 5$ /issue

AJUR Subscriptions

Physics 205

University of Northern lowa

Cedar Falls, lowa 50614-0150 USA

Cheques payable to "AJUR"

Institutions will be invoiced. 


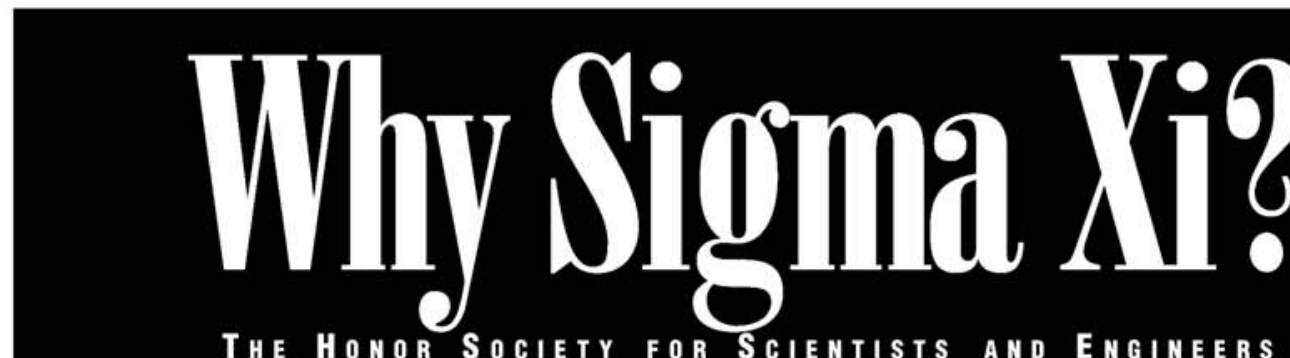

ThE HONOR SOCIETY FOR SCIENTISTS AND ENGINEER

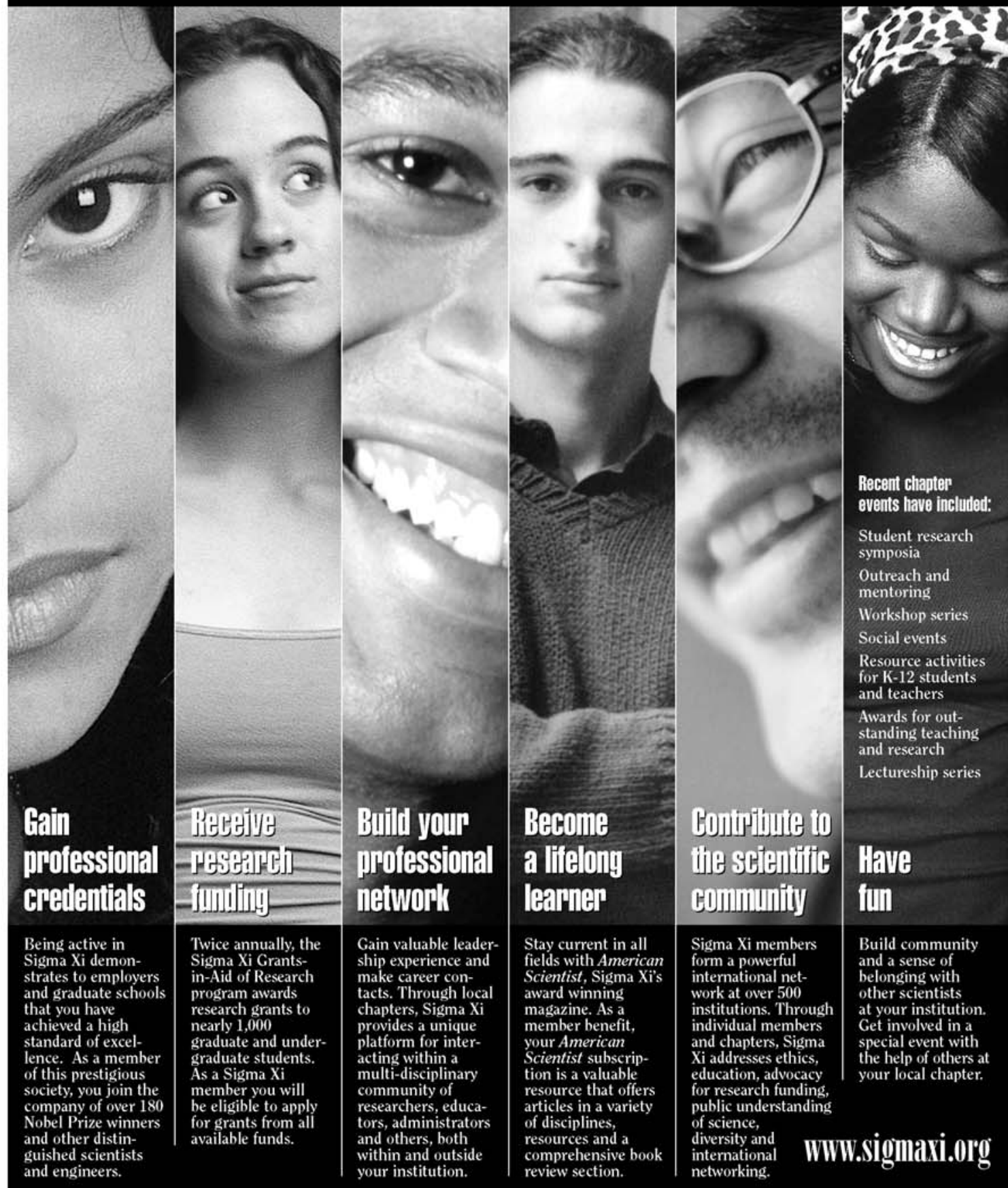

Sigma Xi, The Seientific Research Society • 98 Alexander Drive • P.O. Box 13975 • Research Triangle Park, NC 27708 • 818-549-4691 • 800-243-6534 